\title{
ALMOST SPLIT SEQUENCES FOR GROUP ALGEBRAS OF FINITE REPRESENTATION TYPE
}

BY

\author{
IDUN REITEN
}

\begin{abstract}
ABSTRACr. Let $k$ be an algebraically closed field of characteristic $p$ and $G$ a finite group such that $p$ divides the order of $G$. We compute all almost split sequences over $k G$ when $k G$ is of finite representation type, or more generally, for a finite dimensional $k$-algebra $\Lambda$ given by a Brauer tree. We apply this to show that if $\Lambda$ and $\Lambda^{\prime}$ are stably equivalent $k$-algebras given by Brauer trees, then they have the same number of simple modules.
\end{abstract}

Introduction. Let $\Lambda$ be an artin algebra, that is, an artin ring which is finitely generated as a module over its center. Denote by $\bmod \Lambda$ the category of finitely generated (left) $\Lambda$-modules. We recall from [5] that a nonsplit exact sequence $0 \rightarrow A \stackrel{f}{\rightarrow} B \stackrel{g}{\rightarrow} C \rightarrow 0$ in $\bmod \Lambda$ is said to be almost split if $A$ and $C$ are indecomposable, and given any morphism $h: X \rightarrow C$ which is not a splittable epimorphism, there is a morphism $s: X \rightarrow B$ such that $g s=h$. Given an indecomposable nonprojective $C$ in $\bmod \Lambda$ (or an indecomposable noninjective $A$ in $\bmod \Lambda$ ), we have existence and uniqueness of an almost split sequence $0 \rightarrow A \stackrel{f}{\rightarrow} B \stackrel{g}{\rightarrow} C \rightarrow 0$ [5]. A further study of the invariants determined by almost split sequences, for example the number of summands in a decomposition of the middle term $B$ as a direct sum of indecomposable modules, is made in [6], and some methods for computing almost split sequences are discussed in [7]. Since the structure of almost split sequences is closely connected to the representation theory of the ring, it is of interest to obtain as much information as possible about them.

In this paper we describe all almost split sequences for a particular class of finite dimensional $k$-algebras $\Lambda$, where $k$ throughout this paper will denote an algebraically closed field; namely algebras given by Brauer trees (see [9]).

We say that $\Lambda$ is given by a Brauer tree if the following conditions hold: Let $P_{1}, \ldots, P_{e}$ denote the nonisomorphic indecomposable projective $\Lambda$-modules, and $S_{1}, \ldots, S_{e}$ the nonisomorphic simple modules, where $S_{i} \simeq P_{i} / \mathrm{r} P_{i}$. Here $\mathfrak{r}$ denotes the radical of $\Lambda$. There is a tree with $e$ edges, which are in one-one correspondence with the indecomposable projective, and hence also

Received by the editors March 30, 1976.

AMS (MOS) subject classifications (1970). Primary 16A26, 16A46, 16A64; Secondary 18 G05. 
with the simple, $\Lambda$-modules, and $e+1$ vertices, one of which may be exceptional. In that case there is assigned a number $m>1$ to this vertex. Further there is for each vertex a circular ordering of the edges containing this vertex. We have $\mathfrak{r} P_{i}=P_{i, 1}+P_{i, 2}$ where $P_{i, 1}$ and $P_{i, 2}$ are uniserial modules (i.e., have unique composition series), and $P_{i, 1} \cap P_{i, 2}=\operatorname{soc} P_{i} \cong S_{i}$. Denote by $E_{i}$ the edge corresponding to $P_{i}$ in the tree, and by $E_{i, 1}$ and $E_{i, 2}$ the vertices which are the endpoints of $E$. Then the composition factors of $P_{i, j}, j=1,2$, are determined, from top to bottom, by the edges in the circular ordering of the edges containing $E_{i, j}$, in such a way that we start with the simple module corresponding to the edge following $E$, and end at the simple module corresponding to $E$, by going around once, except when $E_{i, j}$ is exceptional. Then we go around $m$ times.

The structure of the indecomposable finitely generated modules over $k$-algebras given by Brauer trees was given in [9], [10]. In particular, this gives a description of the indecomposable finitely generated $k G$-modules, where $G$ is a finite group and $k$ has characteristic $p$ dividing the order of $G$, and such that $k G$ is of finite representation type [9], [10]. We recall some of the results from [9] and [10] in $\$ 1$, and give some easy consequences which will be needed in the rest of the paper. Another description of the indecomposable finitely generated $\Lambda$-modules is given in [8].

In $\$ 2$ we describe indecomposable submodules and indecomposable factor modules of indecomposable modules over a $k$-algebra $\Lambda$ given by a Brauer tree, and use this description to compute all almost split sequences in mod $\Lambda$.

We recall that two artin algebras $\Lambda$ and $\Lambda^{\prime}$ are said to be stably equivalent if the categories $\bmod \Lambda$ and $\bmod \Lambda^{\prime}$ modulo projectives are equivalent categories (see [1], [2]). It is an open problem in general whether two stably equivalent artin algebras have the same number of nonprojective simple modules. In $\$ 3$ we propose a method of using almost split sequences to get more information about this problem. We illıstrate our method by using the computations from $\S 2$ to show that if $\Lambda$ and $\Lambda^{\prime}$ are stably equivalent $k$-algebras given by Brauer trees, then they have the same number of nonprojective simple modules.

The results in this paper were announced at the Workshop on Permutation Groups and Indecomposable Modules in Giessen, September 1975.

1. Preliminaries. Let $k$ be an algebraically closed field and $\Lambda$ a (finite dimensional) $k$-algebra given by a Brauer tree. In this section we shall recall from [9], [10] what the indecomposable $\Lambda$-modules look like, and give some elementary properties about them.

Let $P_{1}, \ldots, P_{e}$ denote the indecomposable projective $\Lambda$-modules, $S_{1}, \ldots, S_{e}$ the corresponding simple modules. We have $\mathfrak{r} P_{i}=P_{i, 1}+P_{i, 2}$, where $P_{i, 1}$ and $P_{i, 2}$ are uniserial and $P_{i, 1} \cap P_{i, 2}=\operatorname{soc} P_{i} \cong P_{i} / \mathrm{r} P_{i}$. From the 
connection with the tree it is easy to see that $P_{i, 1} / \operatorname{soc} P_{i}$ and $P_{i, 2} / \operatorname{soc} P_{i}$ have no composition factors in common. A nonzero module $M_{i}$ is said to be elementary if $M_{i}$ is a proper factor module of some indecomposable projective $\Lambda$-module. If $M_{i}$ is elementary, we have $\mathrm{r} M_{i}=M_{i, 1} \amalg M_{i, 2}$, where $M_{i, 1}$ and $M_{i, 2}$ are uniserial (or zero). Two nonzero modules $M$ and $N$ are said to be complementary if there is some indecomposable projective $\Lambda$-module $P_{i}$ such that there are monomorphisms $M \rightarrow P_{i, 1}$ and $N \rightarrow P_{i, 2}$.

An indecomposable nonprojective $\Lambda$-module $M$ is completely determined, up to reversal of order, by a sequence $M_{1}, \ldots, M_{t}$ of elementary submodules of $M$ which generate $M$ and such that

(i) $\left(M_{1}+\cdots+M_{j}\right) \cap\left(M_{j+1}+\cdots+M_{t}\right)=\operatorname{soc} M_{j, 2}=\operatorname{soc} M_{j+1,1} \neq$ (0), $1<j<t$, for appropriate choice of decomposition $\mathrm{r} M_{i}=M_{i, 1} \amalg M_{i, 2}, 1<$ $i<t$.

(ii) $M_{j} / M_{j, 1}$ and $M_{j+1} / M_{j+1,2}$ are complementary for $1<j<t$. In this case we write $M=\left(M_{1}, \ldots, M_{t}\right)$. The $M_{i}$ are completely determined by $M$. (If $t=1$ and $M_{1}$ is uniserial, we choose the notation such that $M_{1,2}$ is zero.)

If conversely we are given a sequence $M_{1}, \ldots, M_{t}$ of elementary modules, where soc $M_{j, 2} \cong \operatorname{soc} M_{j+1,1} \cong T_{j}$ and $M_{j} / M_{j, 1}$ and $M_{j+1} / M_{j+1,2}$ are complementary; $1<j<t$, there is associated with the sequence $M_{1}, \ldots, M_{t}$ an indecomposable nonprojective $\Lambda$-module $M=\left(M_{1}, \ldots, M_{t}\right)$, of which the $M_{i}$ can be considered submodules in a natural way. $M$ is constructed as follows. For each $i=1, \ldots, t-1$, let $\psi_{i, 1}: T_{i} \rightarrow M_{i, 2}$ and $\psi_{i, 2}: T_{i} \rightarrow M_{i+1,1}$ be nonzero morphisms. Let $X$ be the submodule of $M_{1} \amalg \cdots \amalg M_{t}$ whose elements are

$$
\begin{aligned}
\left\{\left(\psi_{1,1}\left(x_{1}\right), \psi_{1,2}\left(x_{1}\right)+\right.\right. & \psi_{2,1}\left(x_{2}\right), \ldots, \\
& \left.\left.\psi_{t-2,2}\left(x_{t-2}\right)+\psi_{t-1,1}\left(x_{t-1}\right), \psi_{t-1,2}\left(x_{t-1}\right)\right), x_{i} \in T_{i}\right\} .
\end{aligned}
$$

Then $M=M_{1} \amalg \cdots \amalg M_{t} / X$.

To see the elementary properties of the indecomposable $\Lambda$-modules which we state at the end of this section, it is useful to have in mind the close connection between indecomposable modules and paths in the associated tree [9]. We refer to [9] for the details on this, and indicate here only briefly how a path in the tree is associated with an indecomposable $\Lambda$-module. Let $M=$ $\left(M_{1}, \ldots, M_{t}\right)$ be an indecomposable nonprojective $\Lambda$-module, and consider the following sequence of simple $\Lambda$-modules: $\operatorname{soc} M_{1,1}$ (if $M_{1,1} \neq(0)$ ), $M_{1} / \mathrm{r} M_{1}$, soc $M_{1,2}, \quad M_{2} / \mathrm{r} M_{2}$, soc $M_{2,2}, \ldots, M_{t-1} / \mathrm{r} M_{t-1}$, soc $M_{t-1,2}$, $M_{t} / \mathfrak{r} M_{t}$, soc $M_{t, 2}$ (if $M_{t, 2} \neq(0)$ ). Then the associated sequence of edges $E_{1}, \ldots, E_{s}$ constitutes a path in the Brauer tree. These edges are either all distinct, or there is some $i$ such that $E_{i}=E_{i+1}$. Then $E_{i}$ and $E_{i+1}$ have the exceptional vertex in common, and this vertex is not a vertex of any of the 
other $E_{j}$. Further $E_{1}, \ldots, E_{i}$ and $E_{i+1}, \ldots, E_{s}$ are distinct. We observe that every second edge in such a path corresponds to a composition factor in $M / \mathfrak{r} M$ and every second edge to a composition factor in soc $M$. In particular, $M / \mathrm{r} M$ and soc $M$ have no repeated composition factors. It is also possible to conclude that at most one $M_{i, j}$ has repeated composition factors.

We shall now give some easy consequences of the descriptions of indecomposable $\Lambda$-modules given in [9], [10]. The essential properties needed to prove these results have been recalled above. In addition we freely use the results given in [9], [10].

LEMMA 1.1. Let $M=\left(M_{1}, \ldots, M_{t}\right)$ be an indecomposable nonprojective $\Lambda$-module. If $U$ is a nonsimple uniserial submodule of $M$, then $U$ is contained in a unique $M_{i}$.

Proof. The submodules $M_{i, 2}+M_{i+1,1}, 1<i<t$, of $M$ are submodules of indecomposable projective modules, and $\mathrm{r} M$ is a direct sum of these modules and $M_{1,1}$ and $M_{t, 2}$. Since there are no repeated composition factors in soc $M$, these are the unique maximal indecomposable submodules of $\mathrm{r} M$ with given simple socle, and any uniserial submodule of $M_{i, 2}+M_{i+1,2}$ is contained in $M_{i, 2}$ or $M_{i+1,1}$. Hence we are done if $U \subseteq \mathrm{r} M$. If $U \& \mathrm{r} M$, then we must have $U=M_{i}$, for some $i$, and this $M_{i}$ must then be uniserial. This finishes the proof of the lemma.

LEMMA 1.2. Let $M=\left(M_{1}, \ldots, M_{t}\right)$ be an indecomposable nonprojective $\Lambda$-module. If $M_{i}$ is not uniserial, then $M_{i}$ is the unique elementary submodule of $M$ having soc $M_{i}$ as socle.

Proof. Let $N$ be an elementary submodule of $M$ such that $\operatorname{soc} N \cong \operatorname{soc} M_{i}$ $\simeq S \amalg T$, where $S$ and $T$ are simple $\Lambda$-modules. Considering the associated tree, there is a unique edge $E$ with corresponding simple module $U$, such that the edges corresponding to $S, U, T$ form a path in the tree. Hence $M_{i} / \mathrm{r} M_{i} \simeq$ $U \simeq N / \mathrm{r} N$. If $N \mathbb{Z} \mathrm{r} M$, it is clear that $N=M_{i}$, since $M / \mathrm{r} M$ has no repeated composition factors. Assume then that $N \subseteq \mathrm{r} M$. We have $\mathrm{r} N=$ $N_{1} \amalg N_{2}$, where $N_{1}$ and $N_{2}$ are uniserial, and, by Lemma $1.1, N_{1}$ and $N_{2}$ are submodules of a unique $M_{j}$, which in each case clearly must be $M_{i}$. It is also clear that one of the vertices of $E$ is exceptional and that the only $M_{j}$ having $U$ as a composition factor must be $M_{i}$. Hence- $N \subseteq \mathfrak{r} M_{i}=M_{i, 1} \amalg M_{i, 2}$, which is impossible. This finishes the proof of the lemma.

Lemma 1.3. Let $U, V_{1}$ and $V_{2}$ be uniserial $\Lambda$-modules, and assume that $U$ and $V_{1}$ and $U$ and $V_{2}$ are complementary. Then there is either a monomorphism $V_{1} \rightarrow V_{2}$ or a monomorphism $V_{2} \rightarrow V_{1}$. 
Proof. Since $U$ and $V_{1}$ are complementary, there is by definition an indecomposable projective $\Lambda$-module $P_{i}$ with monomorphisms $U \rightarrow P_{i, 1}, V_{1} \rightarrow$ $\boldsymbol{P}_{i, 2}$. Similarly, there is an indecomposable projective $\Lambda$-module $\boldsymbol{P}_{j}$ and monomorphisms $U \rightarrow P_{j, 1}, V_{2} \rightarrow P_{j, 2}$, with appropriate choice of notation. Clearly $P_{i} \simeq P_{j}$, and since $P_{i, 2} \simeq P_{j, 2}$ is uniserial, our desired conclusion follows.

2. The almost split sequences. Let $\Lambda$ be a $k$-algebra given by a Brauer tree ( $k$ algebraically closed), and let $M=\left(M_{1}, \ldots, M_{t}\right)$ be an indecomposable nonprojective $\Lambda$-module. To describe the almost split sequences in $\bmod \Lambda$, we shall need to describe the indecomposable submodules of $M$ and the indecomposable modules having $M$ as a factor module. We have the following description.

Proposition 2.1. Let $M=\left(M_{1}, \ldots, M_{t}\right)$ be an indecomposable $\Lambda$-module.

(a) The indecomposable modules with a monomorphism to $M$ are $N=$ $\left(N_{1}, \ldots, N_{s}\right)$, where for some $i>1$ such that $i+s-1<t$, we have $N_{2} \cong$ $M_{i+1}, \ldots, N_{s-1} \cong M_{i+s-2}$ and further $N_{1} \cong M_{i}$ or there is a monomorphism $N_{1} \rightarrow M_{i, 2}$, and $N_{s} \simeq M_{i+1-1}$ or there is a monomorphism $N_{s} \rightarrow M_{i+t-1,1}$.

(b) The indecomposable nonprojective modules with a nonzero morphism onto $M$ are $L=\left(L_{1}, \ldots, L_{r}\right)$, where for some $i>1$ such that $i+t-1<r$, we have $M_{2} \cong L_{i+1}, \ldots, M_{t-1} \cong L_{i+t-2}$, and $M_{1} \cong L_{i} / K$, where $K$ is a submodule of $L_{i, 1}$ which is nonzero if $i>1, M_{t} \cong L_{i+t-1} / K^{\prime}$, where $K^{\prime}$ is a submodule of $L_{i+t-1,2}$ which is nonzero if $i+t-1<r$.

Proof. (a) Let $N=\left(N_{1}, \ldots, N_{s}\right)$ be an indecomposable $\Lambda$-module where for some $i>1$ such that $i+s-1<t$, we have $N_{2} \simeq M_{i+1}, \ldots, N_{s-1} \simeq$ $M_{i+s-2}$ and further $N_{1} \cong M_{1}$ or there is a monomorphism $N_{1} \rightarrow M_{i, 2}$, and $N_{s} \cong M_{i+t-1}$ or there is a monomorphism $N_{s} \rightarrow M_{i+t-1,1}$. We get a monomorphism $i: N_{1} \amalg \cdots \amalg N_{s} \rightarrow M_{1} \amalg \cdots \amalg M_{t}$ induced by the above data. From the construction of indecomposable modules described in $\$ 1$, it is easy to see that there is induced a monomorphism $N \rightarrow M$.

Assume conversely that $N=\left(N_{1}, \ldots, N_{s}\right)$ is an indecomposable submodule of $M=\left(M_{1}, \ldots, M_{t}\right)$. It is clear that soc $N \subseteq$ soc $M$. Consider the paths in the tree described by $M$ and $N$. Every second edge in a path corresponds to a simple module in the socle, and given edge number $i$ and number $i+2$, then edge number $i+1$ is uniquely determined. Hence the path described by $N$ is, except possibly for one edge at each end, a subpath of the path described by $M$. By Lemma 1.2 we further have that if $M_{i}$ is not uniserial, then $M_{i}$ is the unique elementary submodule of $M$ with soc $M_{i}$ as socle. It follows that $N_{2}=M_{i+1}, \ldots, N_{t-1}=M_{i+t-2}, i>1, i+s-1<t$. If $N_{1}$ is not uniserial, then $N_{1}=M_{i}$, and if $N_{1}$ is uniserial, then $N_{1}$ is by Lemma 1.1 contained in a unique $M_{j}$, which clearly must be $M_{i}$. If $N_{1}$ is properly 
contained in $M_{i}$, then $N_{1} \subseteq \mathrm{r} M_{i}$, so that clearly $N_{1} \subseteq M_{i, 2}$. Similarly we conclude that $N_{s}=M_{i+t-1}$ or $N_{s} \subseteq M_{i+t-1,1}$.

(b) Let $M=\left(M_{1}, \ldots, M_{t}\right)$ be an indecomposable $\Lambda$-module, and assume that $L=\left(L_{1}, \ldots, L_{r}\right)$ is an indecomposable nonprojective $\Lambda$-module where for some $i \geqslant 1$ such that $i+t-1<r$ we have $M_{2} \cong L_{i+1}, \ldots, M_{t-1} \cong$ $L_{i+t-2}$, and $M_{1} \cong L_{i} / K$, where $K$ is a submodule of $L_{i, 1}$ which is nonzero if $i>1$, and $M_{t} \cong L_{i+t-1} / K^{\prime}$, where $K^{\prime}$ is a submodule of $L_{i+t-1,2}$ which is nonzero if $i+t-1<r$. From these data there is induced a natural epimorphism $p: L_{1} \amalg \cdots \amalg L_{r} \rightarrow M_{1} \amalg \cdots \amalg M_{t}$. Let $T_{j} \cong \operatorname{soc} L_{j, 2}, 1<j<r, \psi_{j, 1}$ : $T_{j} \rightarrow L_{j, 2}, \psi_{j, 2}: T_{j} \rightarrow L_{j+1,1}$ nonzero morphisms. Consider the composite morphisms $\phi_{j, 1}: T_{j} \rightarrow L_{j, 2} \rightarrow M_{j-i+1,2}$ and $\phi_{j, 2}: T_{j} \rightarrow L_{j+1,1} \rightarrow M_{j-i+2,1}, i<j<t$ $-2+i$. The submodule $X^{\prime}$ of $L_{1} \amalg \cdots \amalg L_{r}$ is generated by elements $\psi_{j, 1}\left(x_{j}\right)$ $+\psi_{j, 2}\left(x_{j}\right)$, for $x_{j} \in T_{j}, 1<j<r$, and the submodule $X$ of $M_{1} \amalg \cdots \amalg M_{t}$ is generated by the elements $\phi_{j, 1}\left(x_{j}\right)+\phi_{j, 2}\left(x_{j}\right)$, for $x_{j} \in T_{j}, i<j<t-2+i$. If $i<j<t-2+i$, then clearly $p\left(\psi_{j, 1}\left(x_{j}\right)+\psi_{j, 2}\left(x_{j}\right)\right)$ is in $X$. If $i>1$, then $p\left(\psi_{i-1,1}\left(x_{i-1}\right)+\psi_{i-1,2}\left(x_{i-1}\right)\right)=0$ since $p\left(L_{i-1}\right)=0$ and $\psi_{i-1,2}\left(x_{i-1}\right)$ is in soc $L_{i, 1}$ and hence in $K$, where $p(K)=0$. Continuing with similar arguments we get $p\left(X^{\prime}\right) \subseteq X$. Hence there is induced an epimorphism $L \rightarrow M$.

Assume conversely that there is an epimorphism $p: L=\left(L_{1}, \ldots, L_{r}\right) \rightarrow M$ $=\left(M_{1}, \ldots, M_{t}\right)$. Then there is induced an epimorphism $L / \mathrm{r} L \rightarrow M / \mathrm{r} M$. By considering the associated paths in the tree, we then see that the path described by $M$ must be a subpath of the path described by $L$, except possibly for one edge at each end. Clearly $M$ is generated by the submodules $p\left(L_{j}\right)$ which are zero or elementary. Assume that, for some $j, p\left(L_{j-1}\right)$ and $p\left(L_{j+1}\right)$ are both not zero. Since not both $L_{j-1,2}$ and $L_{j, 1}$ can have repeated composition factors, proper factor modules of $L_{j-1,2}$ and $L_{j, 1}$ cannot have socle isomorphic to soc $L_{j, 1}$. Similarly proper factor modules of $L_{j, 2}$ and $L_{j+1,1}$ cannot have socles isomorphic to soc $L_{j, 2}$. From this it follows that $L_{j} \rightarrow p\left(L_{j}\right)$ is an isomorphism. Hence there is some $i \geqslant 1$ such that $i+t-1<r$ and $M_{2} \approx L_{i+1}, \ldots, M_{t-1} \cong L_{i+t-2}$. Clearly $p\left(L_{i}\right)=M_{1}$ and $p\left(L_{i+t-1}\right)=M_{t}$, and if $1<j<i$ or $i+t-1<j<r$, then $p\left(L_{j}\right)=0$. If $i>1$, we then have $p\left(L_{i-1}\right)=0$, and since $L_{i-1} \cap L_{i} \neq(0)$, this means that $\operatorname{Ker}\left(p \mid L_{i, 1}\right) \neq(0)$. Similarly we get that $\operatorname{Ker}\left(p \mid L_{i+t-1,2}\right) \neq(0)$ if $i+t-1<r$.

We shall next associate with a given indecomposable nonprojective $\Lambda$-module $M=\left(M_{1}, \ldots, M_{t}\right)$ some special indecomposable submodules and some special indecomposable modules of which $M$ is a factor module.

Let $P_{1} \rightarrow M_{1}$ be a projective cover. We have, where $S_{1} \approx \operatorname{soc} P_{1}, r P_{1} / S_{1}=$ $P_{1,1} / S_{1} \amalg P_{1,2} / S_{1}$, and there are induced epimorphisms $P_{1,1} / S_{1} \rightarrow M_{1,1}$, $P_{1,2} / S_{1} \rightarrow M_{1,2}$. Then there is an elementary module $N_{1}$ with $N_{1,2} \cong M_{1,2}$ and $M_{1,1} \simeq N_{1,1} / S$, where $S$ is a simple submodule of $N_{1,1}$, if and only if the epimorphism $P_{1,1} / S_{1} \rightarrow M_{1,1}$ is not an isomorphism. And if $N_{1}$ exists, it is clearly unique up to isomorphism. If $N_{1}$ exists, we define 


$$
\gamma_{1}(M)=\left\{\begin{array}{c}
\left(N_{1}, M_{2}, \ldots, M_{t}\right) \text { if } I\left(N_{1,1}\right), \text { the injective envelope of } N_{1,1}, \\
\text { is uniserial, } \\
\left(N_{0}, N_{1}, M_{2} \cdots M_{t}\right) \text { if } I\left(N_{1,1}\right) \text { is not uniserial. } \\
\text { Here } N_{0} \text { is uniserial of maximal length with the property } \\
\text { that } N_{0} \text { and } N_{1,1} \text { are complementary. }
\end{array}\right.
$$

Let $P_{t} \rightarrow M_{t}$ be a projective cover. Then we have, where $S_{t} \cong \operatorname{soc} P_{t}, \mathrm{r} P_{t} / S_{t}$ $=P_{t, 1} / S_{t} \amalg P_{t, 2} / S_{t}$. Further there is an elementary $\Lambda$-module $N_{t}$ such that $N_{t, 1} \cong M_{t, 1}$ and $N_{t, 2} / T \cong M_{t, 2}$ for a simple $\Lambda$-module $T$, if and only if the induced epimorphism $P_{t, 2} / S_{t} \rightarrow M_{t, 2}$ is not an isomorphism. $N_{t}$ is unique if it exists. If $N_{t}$ exists, we define

$$
\gamma_{2}(M)=\left\{\begin{array}{cc}
\left(M_{1}, \ldots, M_{t-1}, N_{t}\right) & \text { if } I\left(N_{t, 2}\right) \text { is uniserial, } \\
\left(M_{1}, \ldots, M_{t-1}, N_{t}, N_{t+1}\right) & \text { if } I\left(N_{t, 2}\right) \text { is not uniserial. } \\
\text { Here } N_{t+1} \text { is maximal uniserial with the property } \\
\text { that } N_{t, 2} \text { and } N_{t+1} \text { are complementary. }
\end{array}\right.
$$

We go on to define two particular submodules of an arbitrary indecomposable nonprojective $\Lambda$-module $M=\left(M_{1}, \ldots, M_{t}\right)$ :

$$
\delta_{1}(M)=\left(M_{1,2}, M_{2}, \ldots, M_{t}\right), \quad \delta_{2}(M)=\left(M_{1}, \ldots, M_{t-1}, M_{t, 1}\right) .
$$

The following result shows the importance of the indecomposable modules associated with $M$ defined above.

Proposition 2.2. (a) $\delta_{1}(M)$ and $\delta_{2}(M)$ are zero or indecomposable, and there are natural proper monomorphisms $\delta_{1}(M) \rightarrow M$ and $\delta_{2}(M) \rightarrow M$. Further for any proper indecomposable submodule $N$ of $M, N \rightarrow M$ factors through $\delta_{1}(M)$ $\rightarrow M$ or through $\delta_{2}(M) \rightarrow M$.

(b) If $\gamma_{i}(M)$ exists, $i=1,2$, there is a natural epimorphism $p_{i}: \gamma_{i}(M) \rightarrow M$. And if $h: L \rightarrow M$ is a proper epimorphism and $L$ is indecomposable, then $\gamma_{1}(M)$ or $\gamma_{2}(M)$ exists, and $h: L \rightarrow M$ factors through $p_{1}: \gamma_{1}(M) \rightarrow M$ or through $p_{2}$ : $\gamma_{2}(M) \rightarrow M$.

(c) If $\gamma_{i}(M)$ exists, then $\delta_{i}(M) \rightarrow M$ factors through $\gamma_{i}(M) \rightarrow M, i=1,2$.

Proof. (a) It is clear that $\delta_{i}(M)$ is zero or indecomposable, and it follows from Proposition 2.1 that there is a monomorphism $\delta_{i}(M) \rightarrow M$ for $i=1,2$. Let $N=\left(N_{1}, \ldots, N_{s}\right)$ be an indecomposable proper submodule of $M$. By Proposition 2.1 there is an $i \geqslant 1$ such that $i+s-1<t$, and $N_{2}=$ $M_{i+1}, \ldots, N_{s-1}=M_{i+s-2}, N_{1} \subseteq M_{i}, N_{s} \subseteq M_{i+s-1}$. If $i>1$ it is clear that $N \rightarrow M$ factors through $\delta_{1}(M) \rightarrow M$. This is also clear if $i=1$ and $N_{1} \subseteq$ $M_{1,2}$. If $i=1$ and $N_{1}=M_{1}$, then either $s<t$, or if $s=t$, then $N_{s} \subseteq M_{t, 1}$. It follows that in this case $N \rightarrow M$ factors through $\delta_{2}(M) \rightarrow M$.

(b) $\gamma_{i}(M)$ is clearly indecomposable if it exists, and there is by Proposition 
2.1 a natural epimorphism $p_{i}: \gamma_{i}(M) \rightarrow M$. Assume that there is a proper epimorphism $h: L \rightarrow M$, where $L=\left(L_{1}, \ldots, L_{r}\right)$ is indecomposable. By Proposition 2.1 we then have for some $i>1$ such that $i+t-1<r$ that $M_{2} \cong L_{t+1}, \ldots, M_{t-1} \cong L_{i+t-2}, p\left(L_{i}\right)=M_{1}$ and $p\left(L_{i+t-1}\right)=M_{t}$. We assume first that $L_{i} \rightarrow M_{1}$ is a proper epimorphism; hence $L_{i, 1} \rightarrow M_{1,1}$ is a proper epimorphism. In the above notation, it is then clear that $N_{1}$ exists, and that there is an epimorphism $L_{i, 1} \rightarrow N_{1,1}$. If this epimorphism is proper, we get an epimorphism $L \rightarrow\left(N_{1}, M_{2}, \ldots, M_{t}\right)$ and the same thing holds if $L_{i, 1} \rightarrow$ $N_{1,1}$ is an isomorphism and $i=1$. We point out that there is a monomorphism $\left(N_{1}, M_{2}, \ldots, M_{t}\right) \rightarrow \gamma_{1}(M)$. If $L_{i, 1} \rightarrow N_{1,1}$ is an isomorphism and $i>1$, it is easy to see that $\gamma_{1}(M)=\left(N_{0}, N_{1}, M_{2}, \ldots, M_{t}\right)$, and then we get a morphism $L \rightarrow \gamma_{1}(M)$. For since $L_{i-1} / L_{i-1,1}$ and $L_{i} / L_{i, 2}$ are complementary, and $N_{0}$ and $N_{1} / N_{1,2}$ are complementary, we have by the maximality of $N_{0}$ a monomorphism $L_{i-1} / L_{i-1,2} \rightarrow N_{0}$. (See Lemma 1.3.) Hence we have gotten a morphism $L \rightarrow \gamma_{1}(M)$ in the case that $L_{i, 1} \rightarrow M_{1,1}$ is a proper epimorphism. If $L_{i, 1} \rightarrow M_{1,1}$ is an isomorphism, then we know that $i=1$. Since $h: L \rightarrow M$ is a proper epimorphism, we must then have that $L_{i+t-1,2} \rightarrow M_{t, 2}$ is a proper epimorphism, and we can then get a morphism $L \rightarrow \gamma_{2}(M)$. Hence we have a composite morphism $g: L \rightarrow \gamma_{i}(M) \rightarrow M$ for $i=1$ or 2 . It is easy to see that $\operatorname{Ker} g=\operatorname{Ker} h$, and since the ring of isomorphisms of an indecomposable module is isomorphic to the field $k$, we get our desired result.

(c) Assume that $\gamma_{1}(M)$ exists. Since $N_{1,2} \cong M_{1,2}$, it is clear that $\delta_{1}(M) \rightarrow M$ factors through $\gamma_{1}(M) \rightarrow M$, and similarly, if $\gamma_{2}(M)$ exists, $\delta_{2}(M) \rightarrow M$ factors through $\gamma_{2}(M) \rightarrow M$.

Define now $e_{i}(M)$ to be $\gamma_{i}(M)$ if $\gamma_{i}(M)$ exists, and $\delta_{i}(M)$ otherwise, $i=1,2$, and let $e(M)=e_{1}(M) \amalg e_{2}(M)$. Let $j_{i}: e_{i}(M) \rightarrow M$ be the natural epimorphism or the natural monomorphism, and consider the morphism $j=\left(j_{1} j_{2}\right): e(M) \rightarrow M$. We have the following useful consequence of the above computations.

COROLlaRY 2.3. Let $M$ be an indecomposable nonprojective $\Lambda$-module, and let $h: X \rightarrow M$ be a morphism which is not an isomorphism and where $X$ is an indecomposable nonprojective $\Lambda$-module. Then there is some morphism s: $X \rightarrow$ $\boldsymbol{e}(M)$ such that $j s=h$.

This result will now enable us to compute the almost split sequences in $\bmod \Lambda$. For this we first recall from [6] that the middle term $B$ in an almost split sequence $0 \rightarrow A \rightarrow B \rightarrow C \rightarrow 0$ over a self-injective algebra has a nonzero projective summand if and only if $C \cong P /$ soc $P$, for an indecomposable nonsimple projective module $P$, and in this case the almost split sequence is isomorphic to the sequence $0 \rightarrow \mathfrak{r} P \rightarrow \mathfrak{r} P / \operatorname{soc} P \amalg P \rightarrow P / \operatorname{soc} P \rightarrow 0$.

To be able to write down the other almost split sequences, we shall need the following notation. Let $M=\left(M_{1}, \ldots, M_{\imath}\right)$ be an indecomposable non- 
projective $\Lambda$-module, and assume that $M$ is not isomorphic to any $P / \operatorname{soc} P$, for an indecomposable projective $\Lambda$-module $P$. Then we define the following:

$$
v(M)=\left\{\begin{array}{l}
\left(N_{0}, N_{1}, M_{2}, \ldots, M_{t-1}, N_{t}, N_{t+1}\right) \text { if } \gamma_{1}(M) \text { and } \gamma_{2}(M) \text { exist, } \\
\left(N_{0}, N_{1}, M_{2}, \ldots, M_{t-1}, M_{t, 1}\right) \\
\text { if } \gamma_{1}(M) \text { exists and } \gamma_{2}(M) \text { does not exist, } \\
\left(M_{1,2} M_{2}, \ldots, M_{t-1}, N_{t}, N_{t+1}\right) \\
\text { if } \gamma_{2}(M) \text { exists and } \gamma_{1}(M) \text { does not exist, } \\
\left(M_{1,2}, M_{2}, \ldots, M_{t-1}, M_{t, 1}\right) \text { if neither } \gamma_{1}(M) \text { nor } \gamma_{2}(M) \text { exists. }
\end{array}\right.
$$

By our assumption on $M$ it cannot happen that $t=1$ and $\gamma_{1}(M)$ and $\gamma_{2}(M)$ do not exist. Hence it is easy to see that $v(M)$ is indecomposable.

We are now in the position to state our main result.

THEOREM 2.4. Let $M=\left(M_{1}, \ldots, M_{t}\right)$ be an indecomposable nonprojective $\Lambda$-module, where $\Lambda$ is a $k$-algebra given by a Brauer tree.

(a) If $M \simeq P /$ soc $P$ for an indecomposable projective $\Lambda$-module $P$, we have an almost split sequence $0 \rightarrow \mathrm{r} P \rightarrow \mathrm{r} P / \operatorname{soc} P \amalg P \rightarrow P / \operatorname{soc} P \rightarrow 0$.

(b) If $M$ is not isomorphic to any $P / \operatorname{soc} P$ for an indecomposable projective $\Lambda$-module $P$, we have an almost split sequence $0 \rightarrow v(M) \rightarrow e(M) \stackrel{j}{\rightarrow} M \rightarrow 0$.

PRoof. (a) follows, as already remarked, from [6, Proposition 4.11].

(b) There is a natural epimorphism or a natural monomorphism $h_{i}$ : $v(M) \rightarrow e_{i}(M)$. Let $h=\left(h_{1},-h_{2}\right): v(M) \rightarrow e(M)$. We claim that we have an exact sequence $0 \rightarrow v(M) \stackrel{h}{\rightarrow} e(M) \stackrel{\dot{m}}{\rightarrow} M \rightarrow 0$. It is easy to see that $j$ is an epimorphism and $h$ is a monomorphism. Further it is not hard to see that $j h$ is zero, and then the exactness follows from a length argument.

Let now $h: X \rightarrow M$ be a morphism, where $X$ is indecomposable. If $X$ is projective, there is some $s: X \rightarrow e(M)$ such that $j s=h$, and if $X$ is not projective, the same conclusion follows from Corollary 2.3. Since $v(M)$ is indecomposable, we can now conclude that $0 \rightarrow v(M) \stackrel{h}{\rightarrow} e(M) \stackrel{\dot{m}}{\rightarrow} M \rightarrow 0$ is an almost split sequence [5]. This finishes the proof of the theorem.

3. An application to stable equivalence. We recall that two artin algebras $\Lambda$ and $\Lambda^{\prime}$ are said to be stably equivalent if the categories modulo projectives $\bmod \Lambda$ and $\bmod \Lambda^{\prime}$ are equivalent categories [1], [2]. It is in general an open question whether two stably equivalent artin algebras have the same number of nonprojective simple modules, even though the answer is known to be positive in certain cases, for example if $\Lambda$ is stably equivalent to a hereditary algebra [4], in particular if $\mathfrak{r}^{2}=0$ [3], or if $\Lambda$ has no nonzero projective injective modules [1].

Here we propose a new method for studying this problem, using almost split sequences. We shall illustrate our method by using our computations of 
almost split sequences in the previous section to show thai two stably equivalent $k$-algebras given by Brauer trees have the same number of nonprojective simple modules.

We start by explaining the basis for our method. Let $\Lambda$ and $\Lambda^{\prime}$ be stably equivalent artin algebras, and let $0 \rightarrow A \rightarrow B \amalg P \rightarrow C \rightarrow 0$ be an almost split sequence of $\Lambda$-modules, where $P$ is projective and $B$ has no nonzero projective summands. Then we know from our methods for computing almost split sequences [7] that there is an almost split sequence $0 \rightarrow K \rightarrow B^{\prime} \amalg Q \rightarrow C^{\prime} \rightarrow 0$ of $\Lambda^{\prime}$ modules, where $Q$ is projective and $B^{\prime}$ corresponds to $B$ and $C^{\prime}$ corresponds to $C$ in the correspondence between the finitely generated modules with no nonzero projective summands induced by the stable equivalence. In particular, if $B=\amalg_{i=1}^{n} B_{i}$ and $B^{\prime}=\amalg_{j=1}^{m} B_{j}^{\prime}$ are decompositions into indecomposable summands, then $n=m$.

For $n \geqslant 0$, denote by $\lambda(n)\left(\lambda^{\prime}(n)\right)$ the number of almost split sequences in $\bmod \Lambda\left(\bmod \Lambda^{\prime}\right)$ such that in a decomposition of the middle term into indecomposable summands, there are exactly $n$ nonprojective summands. Then the above discussion shows that $\lambda(n)=\lambda^{\prime}(n)$ for all $n>0$. If for some class of algebras the number of nonprojective simple $\Lambda$-modules can be expressed in terms of some $\lambda(n)$, then two stably equivalent algebras from this class will have the same number of nonprojective simple modules.

Let $\Lambda$ be a $k$-algebra ( $k$ algebraically closed) given by a Brauer tree. If there is an indecomposable projective $\Lambda$-module of length 2 , it is easy to see that $\Lambda$ is local of Loewy length 2 . The we know that if $\Lambda^{\prime}$ is stably equivalent to $\Lambda$, then $\Lambda^{\prime}$ has also exactly one nonprojective simple module. Hence we can assume that each indecomposable projective $\Lambda$-module has length at least 3. We shall here be interested in the number $\lambda(1)$, and we have the following key result.

Proposition 3.1. Let $\Lambda$ be a k-algebra given by a Brauer tree of Loewy length at least 3. In an almost split sequence $0 \rightarrow A \rightarrow B \rightarrow M \rightarrow 0$, there is exactly one nonprojective summand in a decomposition of $B$ into indecomposable summands, if and only if $M \cong P / \operatorname{soc} P$ or $M \cong P / \mathfrak{r} P$ for an indecomposable projective uniserial module $P$ or $M \cong P_{i} / P_{i, j}, j=1,2$, for an indecomposable projective $\Lambda$-module $P_{i}$ which is not uniserial.

Proof. If $M \cong P /$ soc $P$, where $P$ is indecomposable projective, we know that $B \cong \mathfrak{r} P / \operatorname{soc} P \amalg P$. Hence there is exactly one nonprojective summand in a decomposition of $B$ into indecomposable summands if and only if $P$ is uniserial.

Assume now that $M$ is not isomorphic to any $P / \operatorname{soc} P$, where $P$ is indecomposable projective. By Theorem 2.4 the middle term is $e(M)=$ $e_{1}(M) \amalg e_{2}(M)$, where $e_{i}(M)$ is indecomposable nonprojective or zero. We need to investigate when $e_{i}(M)$ is zero. Write $M=\left(M_{1}, \ldots, M_{t}\right)$. If $t>1$, it 
is easy to see that $\delta_{i}(M)$ is not zero, and consequently $e_{i}(M)$ is not zero. Assume now that $t=1$. Then $\delta_{1}(M)=(0)$ if and only if $M_{1,2}=(0)$, which is the case if and only if $M=M_{1}$ is uniserial. If $M=M_{1}$ is uniserial, then $\gamma_{1}(M)$ does not exist if and only if $M_{1} \cong P_{i} / P_{i, 2}$, where $P_{i}$ is indecomposable projective. Further $\delta_{2}(M)=M_{1,1}$ is zero if and only if $M=M_{1}$ is simple. Then $\gamma_{2}(M)$ does not exist if and only if the projective cover of $M$ is uniserial. This means that $e_{2}(M)$ is zero if and only if $M \cong P / \mathrm{r} F$, where $P$ is uniserial indecomposable projective. This finishes the proof of the proposition.

We have the following immediate consequence of the above.

COROLLARY 3.2. Let $\Lambda$ be a $k$-algebra given by a Brauer tree, of Loewy length at least 3. Then the number $e$ of simple (nonprojective) $\Lambda$-modules is equal to $\lambda(1) / 2$.

We can now state the main result of this section.

THEOREM 3.3. Let $\Lambda$ and $\Lambda^{\prime}$ be stably equivalent $k$-algebras given by Brauer trees. Then $\Lambda$ and $\Lambda^{\prime}$ have the same number of simple nonprojective modules.

We end this section by illustrating that our method can be applied without knowing all almost split sequences for the rings under consideration. We shall give a different proof for the result that two stably equivalent algebras with radical square zero have the same number of nonprojective simple modules. We recall that $\Lambda$ and $\Lambda^{\prime}$ are stably equivalent if and only if $\Lambda^{\text {op }}$ and $\Lambda^{\prime o p}$ are stably equivalent [2]. Denote by $\lambda^{\mathrm{op}}(0)$ the number of almost split sequences in mod $\Lambda^{\text {op }}$ with projective middle term. Our desired result will then be a consequence of the following.

Proposition 3.4. Let $\Lambda$ be an artin algebra and assume that $r^{2}=0$. Then the number of simple nonprojective $\Lambda$-modules is equal to $\lambda^{\text {op }}(0)$.

Proof. In [5] we gave a description of almost split sequences $0 \rightarrow A \rightarrow B \rightarrow$ $C \rightarrow 0$ where $B$ is projective. If $r^{2}=0$, this is the case if and only if $A$ is a simple noninjective module. Hence $\lambda^{\mathrm{op}}(0)$ is equal to the number of simple noninjective $\Lambda^{\mathrm{op}}$-modules, which by the duality between $\bmod \Lambda$ and $\bmod \Lambda^{\mathrm{op}}$ is again equal to the number of simple nonprojective $\Lambda$-modules. This finishes the proof of the proposition.

It is hoped that our method can be applied to prove that two stably equivalent artin algebras have the same number of nonprojective simple modules also in more general situations.

\section{REFERENCES}

1. M. Auslander and I. Reiten, Stable equivalence of Artin algebras, Proc. Conf. on Orders, Group Rings and Related Topics (Ohio State Univ., Columbus, Ohio, 1972), Lecture Notes in Math., vol. 353, Springer-Verlag, Berlin and New York, 1973, pp. 8-71. MR 49 \#356. 
2. Stable equivalence of dualizing R-varieties, Advances in Math. 12 (1974), 306-366. MR 49 \#7251.

3. Stable equivalence of dualizing $R$-varieties. III: Dualizing $R$-varieties stably equioalent to hereditary dualizing R-varieties, Advances in Math. 17 (1975), 122-142.

4. hereditary algebras, Advances in Math. 17 (1975), 167-195.

5. $\longrightarrow$ Representation theory of Artin algebras. III: Almost split sequences, Comm. in Algebra 3 (1975), 239-294.

6. Representation theory of Artin algebras. IV: Invariants given by almost split sequences, Comm. Algebra 5 (1977), 443-518.

7. , Representation theory of Artin algebras. V: Methods for computing almost split sequences and irreducible morphisms, Comm. Algebra 5 (1977),519-554.

8. P. Gabriel, Indecomposable representations. II, Symposia Mathematica, Vol. XI (INDAM, Rome, 1971), Academic Press, New York and London, 1973, pp. 81-104. MR 49 \#5132.

9. G. J. Janusz, Indecomposable modules for finite groups, Ann. of Math. (2) 89 (1969), 209-241. MR 39 \#5622.

10. H. Kupisch, Unzerlegbare Moduln endlicher Gruppen mit zyklischer p-Sylow-Gruppe, Math. Z. 108 (1969), 77-104. MR 39 \#2889.

Department of Mathematics, University of Trondheim, Trondheim, NoRWay 